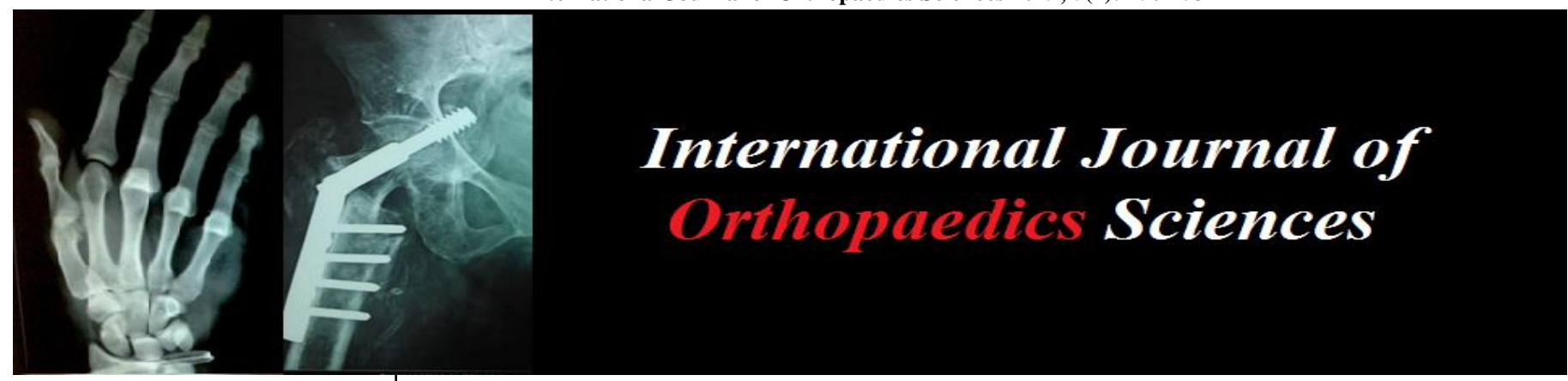

ISSN: $2395-1958$

IJOS 2019; 5(1): 250-253

(C) 2019 IJOS

www.orthopaper.com

Received: 03-11-2018

Accepted: 06-12-2018

Altaf Ahmad Kawoosa

Associate Professor,

Department of Orthopaedics,

Government Hospital for Bone \&

Joint Surgery, Government

Medical College Srinagar,

Jammu and Kashmir, India

Shafeeq Ahmad Sofi

Junior Resident,

Department of Orthopaedics,

Government Hospital for Bone \&

Joint Surgery, Government

Medical College Srinagar,

Jammu and Kashmir, India

Omar Sharief Kirmani

Associate Professor,

Department of Radiodiagnosis

Government Medical College

Srinagar, Jammu and Kashmir,

India
Correspondence

Shafeeq Ahmad Sofi

Junior Resident,

Department of Orthopaedics,

Government Hospital for Bone \&

Joint Surgery, Government

Medical College Srinagar,

Jammu and Kashmir, India

\section{Graft ligamentization after anatomical single bundle ACL reconstruction using hamstring graft}

\author{
Altaf Ahmad Kawoosa, Shafeeq Ahmad Sofi and Omar Sharief Kirmani
}

DOI: https://doi.org/10.22271/ortho.2019.v5.i1e.43

Abstract

Purpose: To assess the ligamentization of the hamstring graft after arthroscopic anatomic single bundle anterior cruciate ligament (ACL) reconstruction using MRI at one year postoperatively.

Patients and Methods: 25 patients (22 males and 03 females) underwent arthroscopic anatomic single bundle ACL reconstruction using hamstring tendon autograft. After ACL reconstruction, the ligamentization of the hamstring graft at one year postoperatively was evaluated on MRI by assessing the signal intensity of the graft and the integration of the graft on the tibial side. The graft integration at the level of the tibial tunnel was evaluated by the presence or absence of synovial fluid at the tunnel-graft interface.

Results: MRI signal intensity of the ACL grafts was low in $13(52 \%)$ cases, intermediate in $10(40 \%)$ cases, and high in $1(4 \%)$ case. Presence of synovial fluid at the graft interface was observed in $3(12 \%)$ cases. The graft ligamentization was present in $23(92 \%)$ cases, as indicated by the Figueroa score of 3-5. Conclusion: The phenomenon of ligamentization of ACL grafts after anatomical ACL reconstruction occurs within 1 year after surgery. MRI is a good tool to evaluate graft integration and graft ligamentization after ACL reconstruction.

Keywords: ACL reconstruction, graft ligamentization, MRI graft signal intensity

\section{Introduction}

After ACL reconstruction, the graft undergoes several phases of healing in the intra-articular graft region and at the site of graft-to-bone incorporation. The changes in the biological and mechanical properties of the healing graft in its intraarticular region are described as the ligamentization process. The biological changes from the time of reconstruction until around the 4th postoperative week is the early graft healing phase, characterized by the graft necrosis, and hypocellularity. The proliferation phase, between the 4th and 12th postoperative week, is characterized by a maximum of cellular activity and changes of the extra-cellular matrix, which are paralleled by the lowest mechanical properties of the reconstructed knee during healing. The ligamentization phase follows directly after the proliferation phase and involves the ongoing process of continuous remodeling of the healing graft toward the morphology and mechanical strength of the intact cruciate.

The appearance of the ACL graft on MRI varies with the type of graft used and with time after graft placement ${ }^{[1,2]}$. In the immediate postoperative period on sagittal MR images, the intraarticular portion of the graft appears as a thick band of homogeneously low signal intensity that extends parallel and posterior to the intercondylar notch ${ }^{[3,4]}$. The tendon grafts undergo a series of biological changes, including initial avascular necrosis, revascularization, cellular repopulation and resynovialization, and finally, remodeling. The amount of revascularization tissue influences the MRI signal intensity of the graft, particularly during the first 2 years postoperatively ${ }^{[5]}$. Between the first 1-3 months after ACL reconstruction, thick proliferating synovial tissue (periligamentous tissue) envelopes the graft and provides its vascular supply ${ }^{[6}$, ${ }^{7]}$. This mass of synovial tissue gradually decreases and becomes a thinner synovial fold that surrounds the ligamentous tissue, after approximately 1 year. The process of gradual transformation of the patellar or hamstring tendon into tissue very similar to the native ACL is referred to as "ligamentization" " ${ }^{7]}$ This process could be indirectly monitored through MRI, as it has been proved that poor biomechanical properties and an incomplete graft maturation are related to a hyperintense graft signal on $\mathrm{MRI}^{[8,9]}$ 


\section{Patients and Methods}

This study was a prospective study conducted at Government Hospital for Bone and Joint Surgery, Srinagar from September 2015 to September 2017, after approval of the institutional board.

The study included 25 patients: 22 males and 3 females, aged between 18-45 years. Informed consent was obtained from all the cases. Arthroscopic anatomic single bundle ACL reconstruction was performed using quadrupled hamstring tendon autograft. MRI evaluation of the operated knees was done at one year postoperatively (Table 1).

Graft ligamentization at the intra-articular portion of graft was assessed by evaluating the signal intensity of the graft and the integration of the graft on the tibial side as described by Figueroa ${ }^{[10]}$ et al. The graft integration at the level of the tibial tunnel was evaluated by the presence or absence of synovial fluid at the tunnel-graft interface.

\section{Figueroa's Score}

\begin{tabular}{|c|c|}
\hline $\begin{array}{c}\text { Graft Integration: Synovial fluid at tunnel-graft } \\
\text { interface }\end{array}$ & Points \\
\hline Positive & 1 \\
\hline Negative & 2 \\
\hline Graft Ligamentization: Graft signal pattern (> 50\%) & \\
\hline Hypointense & 3 \\
\hline Isointense & 2 \\
\hline Hyperintense & 1 \\
\hline
\end{tabular}

The Figueroa score is based on the sum of the points achieved in the 2 items: 2-points represents an insufficiently mature graft, while a score between 3 and 5 points represents a good ligamentization process and graft integration.

MRI signal intensity of graft was classified according to the protocol of Kanamiya ${ }^{[11]}$ et al.

1. Low signal intensity was the same as that of the patellar tendon.

2. Intermediate signal intensity was the same as that of gastrocnemius muscle.

3. High signal intensity was greater than intermediate signal intensity.

\section{Results}

MRI signal intensity of the ACL grafts was low in $13(52 \%)$ cases, intermediate in $10(40 \%)$ cases, and high in $1(4 \%)$ cases. In one patient, graft was invisible and signal intensity was not availible.

Presence of synovial fluid at the interface was observed in 3 $(12 \%)$ cases.

Figueroa score was between 3-5 in 23 (92\%) cases, indicating good ligamentization. In one patient, the ligamentization was absent as indicated by presence of fluid at graft tunnel interface and high signal intensity of the graft.

The MR appearance of the ACL grafts was catagorised into 3 types on the basis of signal intensity and continuity of the ligament, according to Rak's method ${ }^{[13]}$.

Well defined type: the graft was visualized as smooth, continuous band with low intensity over the entire course. This type of graft was observed in 13 (52\%) cases.

Intermediate type: Signal intensity increased and a low signal band was visualized only in part of the graft. This type of graft was observed in $10(40 \%)$ cases.
Indiscernible type: The graft was not identified through the joint cavity due to markedly increased signal intensity. This type of graft was observed in $1(4 \%)$ case. In one patient graft was not visible and signal intensity was not available.
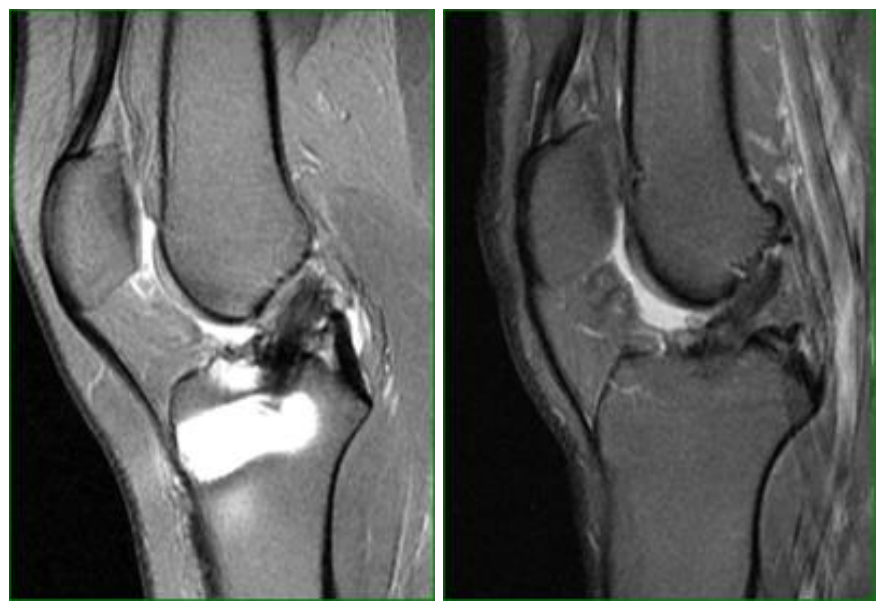

Fig 1: Sagittal MR images 12 months after ACL reconstruction showing the absence of synovial fluid at the bone-tendon graft interface and an hypointense signal of the intra-articular portion of the graft, indicating good ligamentization of ACL graft. (Well defined type grafts)
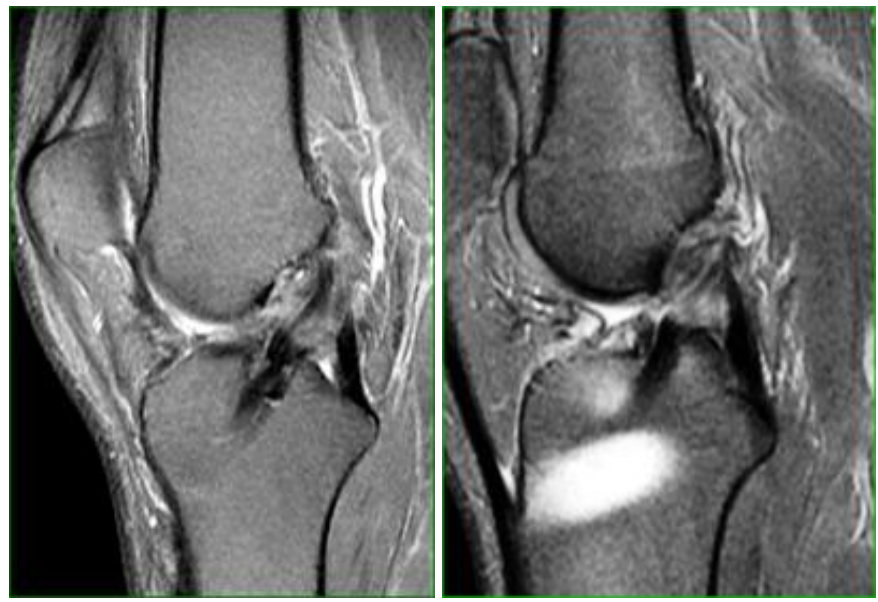

Fig 2: Sagittal MR images 12 months after ACL reconstruction showing intermediate signal intensity within the graft substance due to graft revascularization. (Intermediate type grafts)

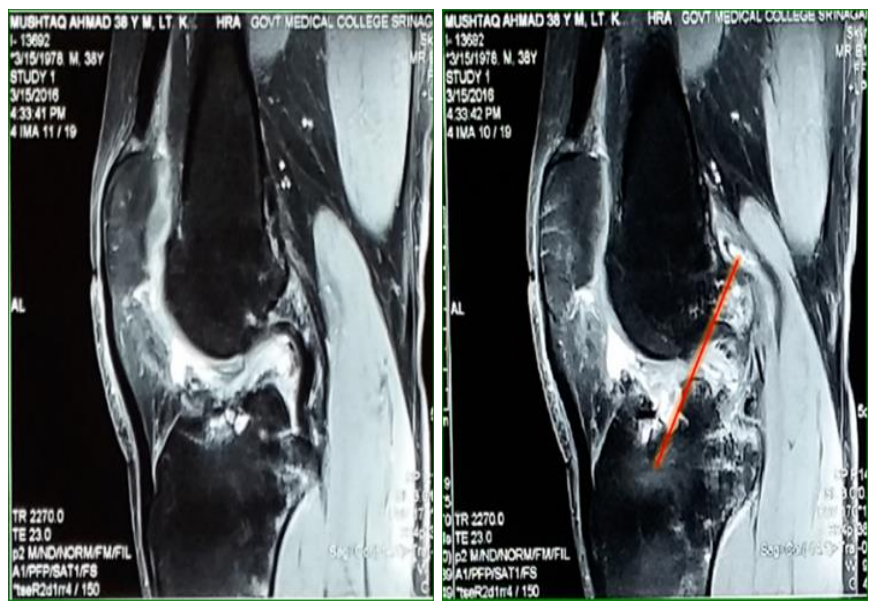

Fig 3: Sagittal MR images of a 38-year-old male 12 months after ACL reconstruction showing hyperintense signal of the acl graft indicating poor ligamentization (Indiscernible type graft). Also the tibial tunnel is found to be located anterior to Blumensatt's line, outside the normal anatomic position. 
Table 1: Post-Operative MRI evaluation

\begin{tabular}{|c|c|c|c|c|c|}
\hline \multirow{2}{*}{ S. No. } & \multirow{2}{*}{ Graft Type on MRI } & \multirow{2}{*}{$\begin{array}{c}\text { MRI Graft } \\
\text { Signal Intensity }\end{array}$} & \multicolumn{3}{|c|}{ Ligamentization } \\
\hline & & & Synovial fluid at graft tunnel interface & Figueroa's Score & Ligamentization \\
\hline 1 & Well defined type & Low & Absent & $3+2=5$ & Good \\
\hline 2 & Intermediate type & Intermediate & Absent & $3+2=5$ & Good \\
\hline 3 & Intermediate type & Intermediate & Absent & $1+2=3$ & Good \\
\hline 4 & Well defined type & Low & Absent & $3+2=5$ & Good \\
\hline 5 & Well defined type & Low & Absent & $3+2=5$ & Good \\
\hline 6 & Indiscernible type & High & Present & $1+1=2$ & INSUFFICIENT \\
\hline 7 & Well defined type & Low & Absent & $1+2=3$ & Good \\
\hline 8 & Intermediate type & Intermediate & Absent & $1+2=3$ & Good \\
\hline 9 & Intermediate type & Intermediate & Absent & $3+2=5$ & Good \\
\hline 10 & Well defined type & Low & Absent & $3+2=5$ & Good \\
\hline 11 & Well defined type & Low & Absent & $3+2=5$ & Good \\
\hline 12 & Intermediate type & Intermediate & Present & $2+1=3$ & Good \\
\hline 13 & Intermediate type & Intermediate & Absent & $3+2=5$ & Good \\
\hline 14 & Intermediate type & Intermediate & Absent & $1+2=3$ & Good \\
\hline 15 & Intermediate type & Intermediate & Absent & $1+2=3$ & Good \\
\hline 16 & Well defined type & Low & Absent & $3+2=5$ & Good \\
\hline 17 & Not available & Not available & Not available & Not available & Not available \\
\hline 18 & Well defined type & Low & Absent & $3+2=5$ & Good \\
\hline 19 & Well defined type & Low & Absent & $3+2=5$ & Good \\
\hline 20 & Intermediate type & Intermediate & Present & $1+2=3$ & Good \\
\hline 21 & Intermediate type & Intermediate & Absent & $3+2=5$ & Good \\
\hline 22 & Well defined type & Low & Absent & $3+2=5$ & Good \\
\hline 23 & Well defined type & Low & Absent & $3+2=5$ & Good \\
\hline 24 & Well defined type & Low & Absent & $3+2=5$ & Good \\
\hline 25 & Well defined type & Low & Absent & $3+2=5$ & Good \\
\hline
\end{tabular}

\section{Discussion}

According to MRI findings in our patients, the graft were categorized as well defined type in $13(52 \%)$ patients, as intermediate type in $10(40 \%)$ patients and as indiscernible type in $1(4 \%)$ patients as per Rak's method. In our study intermediate signal intensity with a low signal band in some portion of graft was found in the grafts of $10(40 \%)$ patients. In these patients, the graft was ligamentized indicated by absence of synovial fluid at the graft tunnel interface. In one patient with with high signal intensity of graft with synovial fluid at the interface, ligamentization was absent. The tibial tunnel was found to be anterior to the tangent of the Blumensatts line.

It has been reported that signal intensity of clinically stable ACL grafts increases up to 12 months after surgery and then decreases over the subsequent 12 months ${ }^{[12]}$. This increase has been attributed to revascularization and cellular infiltration ${ }^{[13]}$ and has been considered an indeterminate finding in the assessment of graft integrity ${ }^{[14]}$.

Jansson et al. ${ }^{[15]}$ reported that during the postoperative phase (12-18 months), the graft may normally show a degree of intrasubstance increased signal intensity on $\mathrm{T} 1$ - and $\mathrm{T} 2$ weighted images that is reflective of synovial and neovascular proliferation around and within the graft, which is referred to as "neoligamentization" of graft tissue. However a long term follow up study conducted by Saupe et al. ${ }^{[16]}$ showed that small amounts of increased intrasubstance graft signal can be seen after anterior cruciate ligament (ACL) reconstruction at long-term follow-up (>4 years) on intermediate-weighted and T2-weighted MR images in approximately two thirds of patients $(70 \%$ and $64 \%$, respectively). Also in a pictorial essay done by Recht and Kramer [17] and a study done by Saupe et al. ${ }^{[16]}$ they reported that T2-weighted images may also show regions of increased signal intensity within an intact graft, if such signal was not iso-intense relative to fluid and not traversing the full thickness of the graft construct. Based on the current literature, the graft SI changes are often seen and probably not clinically significant.

Marumo ${ }^{[18]}$ et al demonstrated that the phenomenon of ligamentization occurs in the successfully reconstructed human ACL within 1 year after surgery. Moreover, they have found that after ACL reconstruction using autografts, the biochemical characteristics of the graft resembled those of the native ACL ${ }^{[18]}$. Falconiero ${ }^{[19]}$ et al concluded that by 12 months after ACL reconstruction with autografts, the graft resembles a normal ACL.

\section{Conclusion}

The phenomenon of ligamentization of ACL grafts after anatomical ACL reconstruction occurs within 1 year after surgery. MRI is a good tool to evaluate graft integration and graft ligamentization after ACL reconstruction.

\section{References}

1. Schatz JA, Potter HG, Rodeo SA et al. MR imaging of anterior cruciate ligament reconstruction. Am J Roentgenol. 1997; 169:223-228.

2. Rak KM, Gillogly SD, Schaefer RA, Yakes WF, Liljedahl RR. Anterior cruciate ligament reconstruction: evaluation with MR imaging. Radiology. 1991; 178:553556.

3. Trattnig S, Rand T, Czerny C et al. Magnetic resonance imaging of the postoperative knee. Top Magn Reson Imaging. 1999; 10:221-23.

4. Howell SM, Berns GS, Farley TE. Unimpinged and impinged anterior cruciate ligament grafts: MR signal intensity measurements. Radiology. 1991a; 179:639-643.

5. Ntoulia A, Papadopoulou F, Zampeli F, Ristanis S, Argyropoulou M, Georgoulis A. Evaluation with contrast-enhanced magnetic resonance imaging of the anterior cruciate ligament graft during its healing process: a two-year prospective study. Skeletal Radiol. 2013; 42:541-552.

6. Yasuda K, Tomiyama Y, Ohkoshi Y, Kaneda K. 
Arthroscopic observations of autogenic quadriceps and patellar tendon grafts after anterior cruciate ligament reconstruction of the knee. Clin Orthop. 1989; 246:217.

7. Amiel D, Kleiner JB, Roux RD et al. The phenomenon of "ligamentization": anterior cruciate ligament reconstruction with autogenous patellar tendon. J Orthop Res. 1986; 4:162-172.

8. Gnannt R, Chhabra A, Theodoropoulos JS, Hodler J, Andreisek G. MR imaging of the postoperative knee. J Magn Reson Imaging. 2011; 34:1007-1021.

9. Naraghi A, White L. MRI evaluation of the postoperative knee: special considerations and pitfalls. Clin Sports Med. 2006; 25:703-725.

10. Figueroa D, Melean P, Calvo R et al. Magnetic resonance imaging evaluation of the integration and maturation of semitendinosus gracilis graft in anterior cruciate ligament reconstruction using autologous platelet concentrate. Arthroscopy. 2010; 26(10):1318-1325.

11. Kanamiya T, Hara M, Naito $M$. Magnetic resonance evaluation of remodeling process in patellar tendon graft. Clin Orthop Relat Res. 2004; 419:202-6.

12. Murakami Y, Sumen Y, Ochi M, Fujimoto E, Adachi N, Ikuta Y. MR evaluation of human anterior cruciate ligament autograft on oblique axial imaging. J Comput Assist Tomogr. 1998; 22:270-275.

13. Yamato M, Yamagishi T. MRI of of patellar tendon anterior cruciate ligament autografts. J Comput Assist Tomogr. 1992; 16:604-607.

14. Cheung Y, Magee TH, Rosenberg ZS, Rose DJ. MRI of anterior cruciate ligament reconstruction. J Comput Assist Tomogr. 1992; 16:134-137

15. Jansson KA, Karjalainen PT, Harilainen A et al. MRI of anterior cruciate ligament repair with patellar and hamstring tendon autografts. Skeletal Radiol 2001; 30:814.

16. Saupe N, White LM, Chiavaras MM, Essue J, Weller I, Kunz M et al. Anterior cruciate ligament reconstruction grafts: MR imaging features at long-term follow-up-correlation with functional and clinical evaluation, Radiology. 2008; 249(2):581-590.

17. Recht MP, Kramer J. MR imaging of the postoperative knee: a pictorial essay, Radiographics: a review publication of the Radiological Society of North America, Inc. 2002; 22(4):765-774.

18. Marumo $\mathrm{K}$, Saito $\mathrm{M}$, Yamagishi $\mathrm{T}$ et al. The ligamentization process in human anterior cruciate ligament reconstruction with autogenous patellar and hamstring tendons: a biochemical study. Am J Sports Med. 2005; 33:1166-73.

19. Falconiero RP, DiStefano VJ, Cook TM. Revascularization and ligamentization of autogenous anterior cruciate ligament grafts in humans. Arthroscopy. 1998; 14:197-205. 